\title{
Optimal viewing position in vertically and horizontally presented Japanese words
}

\author{
NATSUMI KAJII and NAOYUKI OSAKA \\ Kyoto University, Kyoto, Japan
}

\begin{abstract}
In the present study, the optimal viewing position (OVP) phenomenon in Japanese Hiragana was investigated, with special reference to a comparison between the vertical and the horizontal meridians in the visual field. In the first experiment, word recognition scores were determined while the eyes were fixating predetermined locations in vertically and horizontally displayed words. Similar to what has been reported for Roman scripts, OVP curves, which were asymmetric with respect to the beginning of words, were observed in both conditions. However, this asymmetry was less pronounced for vertically than for horizontally displayed words. In the second experiment, the visibility of individual characters within strings was examined for the vertical and horizontal meridians. As for Roman characters, letter identification scores were better in the right than in the left visual field. However, identification scores did not differ between the upper and the lower sides of fixation along the vertical meridian. The results showed that the model proposed by Nazir, O'Regan, and Jacobs (1991) cannot entirely account for the OVP phenomenon. A model in which visual and lexical factors are combined is proposed instead.
\end{abstract}

Word recognition accuracy for briefly presented words strongly depends on the position in the word at which the eye fixates (Brysbaert, Vitu, \& Schroyens, 1996; Deutsch \& Rayner, 1999; Farid \& Grainger, 1996; Nazir, Heller, \& Sussmann, 1992; Nazir, Jacobs, \& O'Regan, 1998; Nazir, O'Regan, \& Jacobs, 1991). Performance is best when the eyes fixate near the word center and decreases as the fixation position deviates from this optimal viewing position (OVP). OVP effects, which indicate the consequences of within-word fixation positions for the efficiency of word recognition performance, have been reported for a variety of dependent measures. Thus, naming latencies and lexical decision times (when the eye is free to move in the word) are shortest when the eye starts to fixate the word near its center (Brysbaert \& d'Y dewalle, 1988; O'Regan \& Jacobs, 1992; O'Regan, Lévy-Schoen, Pynte, \& Brugaillère, 1984), and the percentage of correct identification is highest for fixation at the OVP (Brysbaert et al., 1996; Deutsch \& Rayner, 1999; Farid \& Grainger, 1996; Nazir et al., 1992; Nazir et al., 1998). Eye movement behavior is affected as well: The probability that a word will be fixated only once is maximal when the eye lands near the middle of the word and decreases as the eye's initial fixation position deviates from the OVP (McConkie, Kerr,

This work was supported in part by Grants 09044007 and 0955100 from the Ministry of Education, Japan. We thank Tatjana A. Nazir and the members of the "Vision Lunch meeting" for their careful reading of and suggestions for our work. We also thank John Henderson, Sandy Pollatsek, Keith Rayner, and Françoise Vitu for their invaluable reviews of a previous version of this manuscript. Correspondence concerning this article should be addressed to N. Kajii, Department of Psychology, Graduate School of Letters, Kyoto University, Kyoto 606-8501, Japan (e-mail: kajii@kupsy.kyoto-u.ac.jp).
Reddix, Zola, \& Jacobs, 1989; O’Regan et al., 1984; Radach \& Kempe, 1993; Rayner, Sereno, \& Raney, 1996; Underwood, Clews, \& Everatt, 1990; Vitu, 1991; Vitu, O'Regan, \& Mittau, 1990). In addition, it should be noted that the preferred viewing location (Rayner, 1979), the position within the word at which the eye lands most frequently in reading, is also around the center of the word.

Researchers seem to agree that the major determinant of the OVP phenomenon is the drop-off of visual acuity even within the fovea. The minimal angle of resolution increases nearly as a linear function of the distance from the center of vision (Weymouth, Hines, Acres, Raaf, \& Wheeler, 1928). Recognition performance for words that are displayed at the center of vision should, therefore, be better than performance for words located more peripherally, because the centrally displayed words benefit from higher resolution. In line with this idea, McConkie et al. (1989) proposed a solution of how the drop-off of visual acuity could give rise to the OVP phenomenon. The model proposed by these authors is based on three assumptions: (1) The amount of visual information obtained from a letter decreases as a linear function of its distance from the center of fixation, (2) the total amount of visual information available from a word is the sum of the information available from all of its letters, and (3) the frequency of identifying a word during the initial fixation on it is a linear function of the amount of visual information available from it. Following these assumptions, the inverted-U-shaped function of the OVP curves for word recognition accuracy could be predicted.

This summed letter information model, however, had at least two flaws (Nazir et al., 1991). First, the term visual information was not well defined. Second, the model could not explain why the OVP is frequently observed to 
be slightly to the left, and not at the word's center (Brysbaert et al., 1996; Farid \& Grainger, 1996; Nazir et al., 1992; Nazir et al., 1998; Nazir et al., 1991). Given that the visual information available from a stimulus decreases linearly at the same rate on both the right and the left side of fixation, the theoretical OVP curve should be symmetric, with its peak at the word center, and not to the left of it. To solve these problems, Nazir et al. (1991) proposed a modification of the former model. The term visual information in the original version was replaced by letter visibility, which is obtained empirically by determining the probability of identifying individual letters at different locations within strings. The probability of identifying a word is then calculated by multiplying these individual letter probabilities. On the basis of the fact that the identification of letters in strings is higher in the right than in the left visual field (Bouma, 1973; Bouma \& Legein, 1977), the leftward asymmetry of the empirically observed OVP curves was well explained by this multiplied letter identification probability (MLIP) model (Nazir et al., 1991; see also Nazir et al., 1992; Nazir et al., 1998). It should be noted that the MLIP model assumes no lexical influence.

Subsequent studies showed, however, that the laterality of language and lexical factors could influence the OVP phenomenon. Brysbaert et al. (1996), for instance, suggested that cortical hemispheric differences in the processing of language favors the identification of letters in the right visual field, because this information is directly projected to the language-dominant left hemisphere. Word recognition is therefore better while fixating left of the word center, because more letters fall into the right visual field. Furthermore, the authors reported that the OVP varied, depending on the distribution of lexical information within the stimulus words: The OVP was left of word center for words that were highly predictable from their first trigram, whereas it was almost at word center for words that were more predictable from their last trigram. Moreover, in a cross-linguistic study by Farid and Grainger (1996), it was shown that in Arabic words, the OVP is left of word center for prefixed words, whereas it is right of word center for suffixed words. In French, by contrast, the OVP remains left of word center for both prefixed and suffixed words. The authors speculated that the difference in lexical influence between Arabic and French could be due to differences in the morphological structure of the two languages. The latter was confirmed in a recent study with Hebrew words by Deutsch and Rayner (1999).

In sum, variations in the OVP phenomenon, such as those described above, cannot be predicted from the left/right asymmetries in the visibility of letters or from the lexical information distribution within words alone. The phenomenon seems to be caused by a combination of several factors, including visual factors, reading habits, lexical information distribution within a word, and, possibly, hemispheric differences in the processing of language (see, e.g., Brysbaert et al., 1996). At present, how- ever, the relative extent to which these factors contribute to the phenomenon and the way they combine with each other remain obscure. To clarify these issues, the influence of each factor should be analyzed separately.

In the present study, we investigated the OVP phenomenon in vertically and horizontally displayed words of Japanese Hiragana (Experiment 1). In addition, for a better understanding of the relation between the OVP phenomenon and visual field asymmetries in the visibility of letters, we also determined identification scores for individual characters in meaningless strings for the vertical and the horizontal meridians (Experiment 2). Investigating the OVP phenomenon in the Japanese script has the following advantages. First, it allows us to better describe the visual aspects of the phenomenon. If the inverse Ushape of the OVP curve is caused by the drop in visual acuity, it should be observed in both vertical and horizontal words, because visual acuity decreases as a linear function of the distance from the center of vision for the vertical as well as for the horizontal meridian. However, given that the drop of acuity with eccentricity is stronger in the vertical meridian (Weymouth et al., 1928), the effect of within-word fixation location should be stronger for vertically than for horizontally displayed words. Second, the comparison of the horizontal and the vertical displays allows us to identify possible influences of factors that are related to the dominance of the left hemisphere for language on the OVP phenomenon. If the laterality of language plays a role in the asymmetry of the OVP phenomenon, this asymmetry should be present in horizontally displayed Japanese words but less pronounced or $a b-$ sent in the vertical display condition.

It is important to note that Japanese readers are equally familiar with vertical and horizontal reading (see the next section for details). Possible differences in the two display conditions cannot, therefore, be attributed to reading habits. Moreover, given that the same subjects will perceive the same words in a vertical and a horizontal display, the present paradigm also controls for lexical factors.

\section{JAPANESE WRITING SYSTEM}

For a better understanding of the following study, we will briefly explain some features of the Japanese writing system. The modern Japanese writing system has unique features in both the direction of writing and the nature of the language symbols. It is written/read vertically (from top to bottom) as well as horizontally (from left to right). In general, the direction of writing is selected mainly by the content of the text. For example, newspapers and novels are usually written vertically, whereas scientific materials are written horizontally. Native Japanese, therefore, are familiar with both vertical and horizontal writing/reading.

As for language symbols, the Japanese writing system is a hybrid. There are two types of writing systems, Kanji and Kana. Single characters represent a morpheme in the Kanji system, whereas a single character represents a syl- 
lable in the Kana system. These two systems are usually mixed in a word or in a sentence. Note that we used Japanese Hiragana characters for the two experiments in the present study. Hiragana is one of the two forms of the Kana system and is used to represent grammatical prefixes and suffixes, function words, and some content words (Rayner \& Pollatsek, 1989; Taylor \& Taylor, 1995). A text, however, can be written only with Hiragana characters in principle, and children start with the Hiragana characters in Japanese reading in their early language acquisition stage.

\section{EXPERIMENT 1}

The purpose of Experiment 1 was to determine the characteristics of the OVP phenomenon with respect to recognizing vertically and horizontally displayed Japanese Hiragana words.

\section{Method}

Subjects. Twenty undergraduate/graduate students with normal or corrected-to-normal vision served as subjects. All the subjects were native Japanese speakers and right-handed.

Materials. A total of 100 six-character Japanese words were included in the experiment. All of the words were written in Hiragana.

As Figure 1 shows, each word was divided equally into five zones one fifth the width of the total word length (e.g., 1.2 letters). The center of each zone (to the nearest pixel) was designated as a potential fixation position. One character space, which subtended equal width for any character, corresponded to 16 pixels in width, which was $0.6^{\circ}$ of arc visual angle.

The degree of familiarity with the stimulus words as standardly perceived was assessed with a computer-based questionnaire in which the Hiragana words were visually presented. For each word, the respondent indicated the perceived level of familiarity by referring to one of five numbers: 1 , often seen or heard; 2 , sometimes seen or heard; 3, cannot say yes or no; 4, not often seen or heard; and 5 , rarely seen or heard. The mean familiarity of 100 words over a total of 23 respondents, who were undergraduate/graduate students, was 2.58 , with a standard deviation of 1.38 .

Design. The words were subdivided into five groups of 20 words, and the 20 subjects were subdivided into five groups of 4 , corresponding to the five different fixation zones at which each word group would be presented. The attribution of a particular word group to a particular fixation zone was performed differently for each of the five groups of subjects, following a Latin-square design. In this way, taken over all subjects, data were available for eye fixation in each of the five zones of each word. Words were displayed either vertically or horizontally. Given the limited number of sixcharacter words in Japanese, the subjects saw the same list of words under both display conditions. The order of display conditions was counterbalanced over subjects, corresponding to each of the five subject groups: One half of the subjects in each subject group saw the vertical condition first and then the horizontal condition, and the other half saw the words in the reversed order.

Procedure. The subject sat at a distance of $50 \mathrm{~cm}$ from the computer screen. In each trial, he or she was instructed to fixate on a gap, corresponding in size to one character, between two aligned central fixation lines (see Figure 2). In the vertical condition, the fixation lines were aligned horizontally and set just right and left of the vertical position at which the target word was to appear. In the horizontal condition, the fixation lines were aligned vertically and set just above and below the horizontal position at which the target word was to appear. The fixation lines remained for $500 \mathrm{msec}$ and were replaced by the stimulus word, which was presented for $72 \mathrm{msec}$. The position of the stimulus word relative to the central fixation point was varied from trial to trial so that the subject fixated on one of the five possible positions in a six-character word. Stimulus exposure was terminated with a string of 11 hashmarks that remained for $800 \mathrm{msec}$. The task of the subjects was to identify the stimulus word that they observed on the screen by typing the word in Roman letters. Visual feedback, which appeared at the bottom of the screen with a left-to-right presentation, was made available. The order of presentation was randomized, with a different order for each subject. The subjects were given five practice trials before starting the main experimental session.

\section{Results}

The mean probabilities of correct word identification for the two display conditions are plotted in Figure 3 as a function of fixation positions. An analysis of variance (ANOVA), with subjects as the random factor and display condition and fixation position as within-subjects factors, showed a significant main effect of display condition $[F(1,19)=36.25, p<.001]$. Recognition performance was globally better for horizontal words than for vertical words. The main effect of fixation position $[F(4,76)=$ $28.44, p<.001]$ and the interaction between these two factors $[F(4,76)=7.15, p<.001]$ were also significant.

As can be seen in Figure 3, a viewing position effect was found for both display conditions. The effect of fixation position was significant for the vertical $[F(4,152)=$ $15.70, p<.001]$ and for the horizontal $[F(4,152)=$ $30.36, p<.001]$ display conditions. Both OVP curves described an inverted-U-shaped function with a maximum at the third fixation zone. For both display conditions, better performance was observed when the eyes were fixating the first half of the words (first and second positions), as compared with the second half [fourth and fifth positions; $t(19)=2.30, p<.05$, and $t(19)=7.72, p<.001$, for the vertical and horizontal conditions, respectively].

The significant interaction between display condition and fixation position indicates, however, that the shapes of the two OVP curves are different. Note that the interaction was not due to a constant multiplication factor. The advantage to fixating on the first half of the word is much stronger for the horizontal display condition than for the vertical display condition. When fixating on zone one, two, or three, performance for horizontally displayed words is better than that for vertically displayed words $[F(1,95)=33.71, p<.001, F(1,95)=17.72, p<.001$, and $F(1,95)=13.15, p<.001$, for first, second, and third positions, respectively]. Performance differed only marginally for fixation zone $4[F(1,95)=3.52, p<.1]$, and no significant difference was observed for zone 5. Thus, the results indicate that the two OVP curves differ in shape. The significant interaction between fixations on the first half (first and second positions) versus the second half (fourth and fifth positions) of words and the display condition $[F(1,19)=20.86, p<.001]$ also confirms that the two OVP curves differ in shape. 

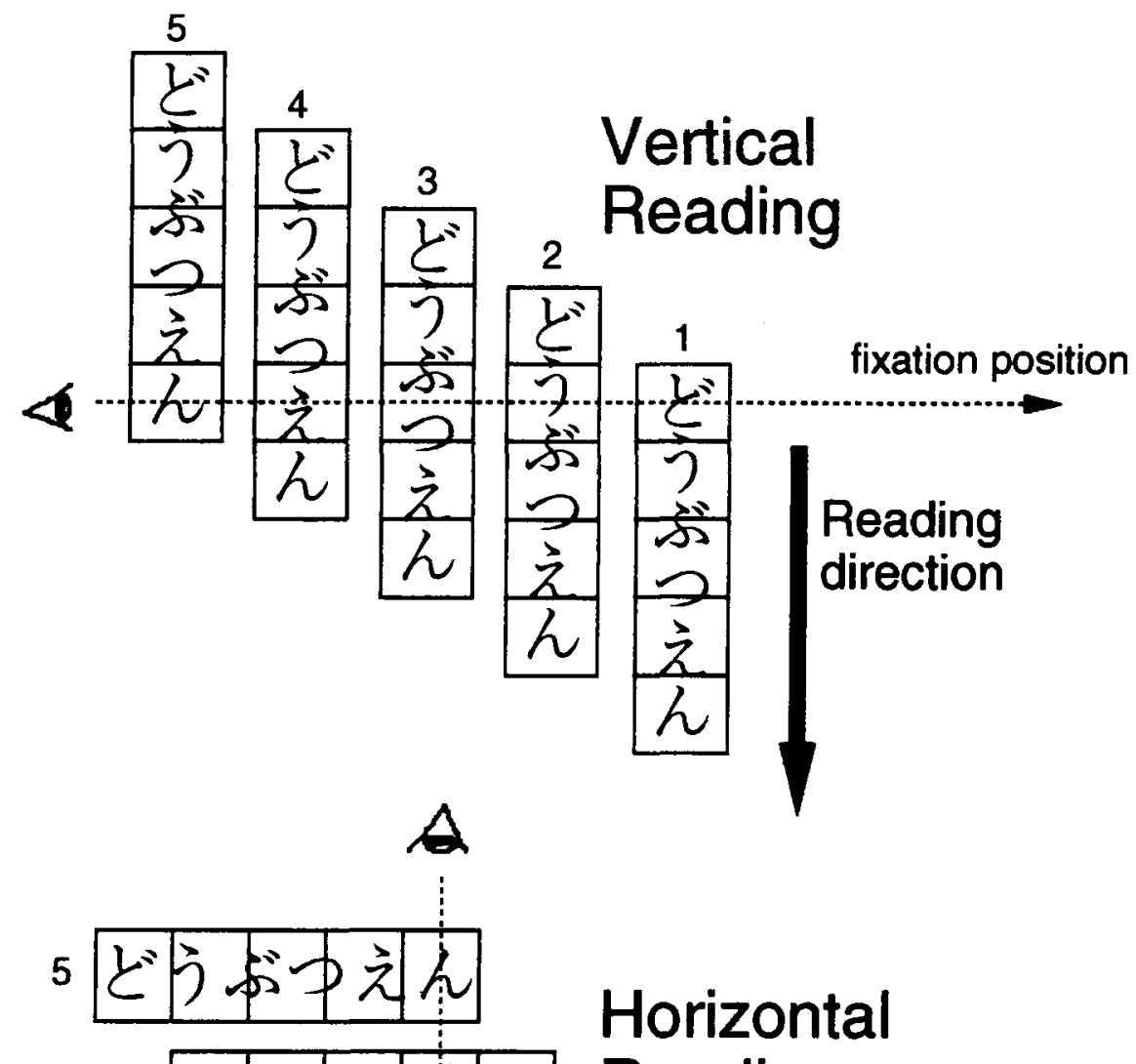

\section{Horizontal Reading}

4
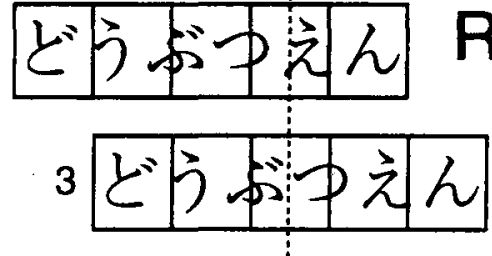

2

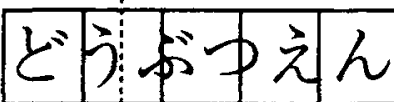

1

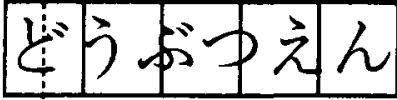

fixation position $\begin{aligned} & \text { Reading } \\ & \text { direction }\end{aligned}$

Figure 1. The five fixation conditions and the horizontal and vertical presentation conditions of stimulus words (Japanese Hiragana). Numerals indicate the fixation position that corresponds to the center of each zone.

\section{Discussion}

Experiment $l$ showed that the OVP phenomenon exists in Japanese Hiragana words for the vertical display condition, as well as for the horizontal. In both cases, the OVP curves were asymmetric to the word beginning. Comparable with what has been reported for Roman script (Brysbaert et al., 1996; Farid \& Grainger, 1996; Nazir et al., 1992; Nazir et al., 1998; Nazir et al., 1991), the OVP in
Japanese words was around the center of the word in the vertical, as well as in the horizontal, display condition. However, the shape of the OVP curve differed, depending on the display condition: The advantage to fixating on the first half of the word was much stronger when the word was displayed horizontally.

The present study is a report of a third non-Roman script, in addition to Arabic (Farid \& Grainger, 1996) and 


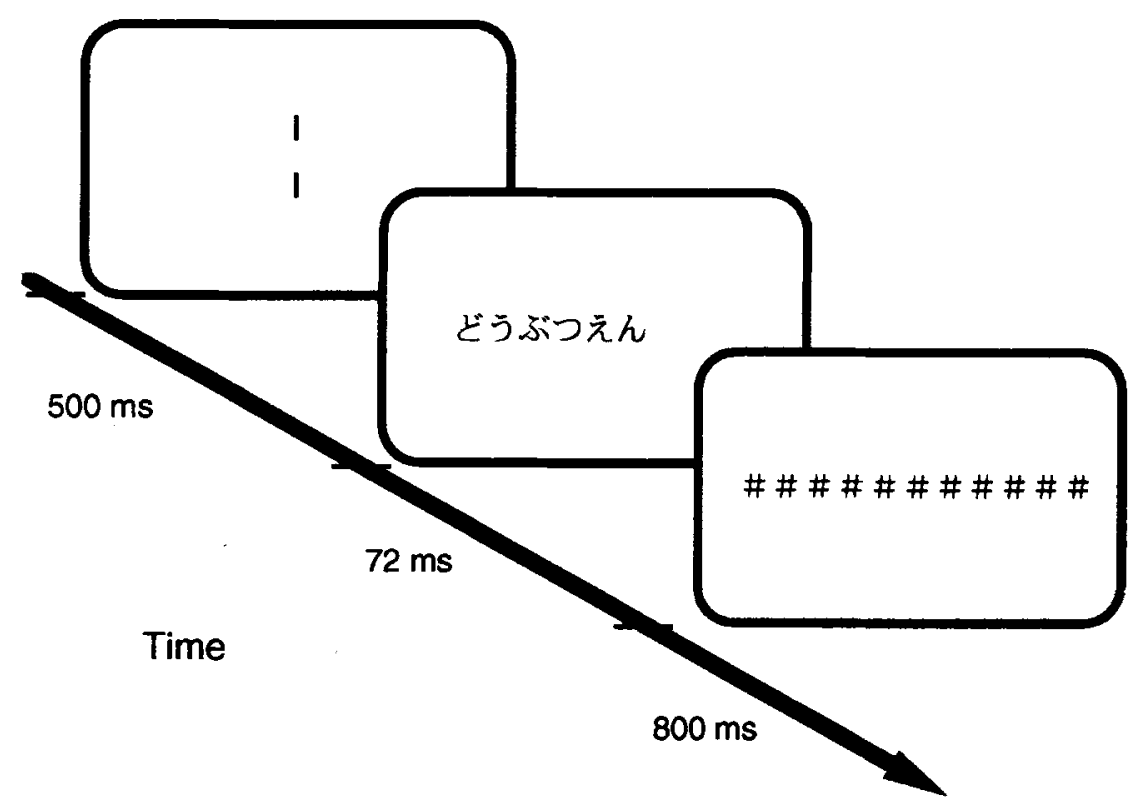

Figure 2. Time course and configuration of the stimulus presentation in Experiment 1.

Hebrew (Deutsch \& Rayner, 1999), for which an OVP phenomenon has been observed. The presence of this phenomenon in four different scripts clearly suggests that the viewing position effect is triggered by some scriptindependent processes of visual word recognition. Given that acuity drops faster along the vertical than along the horizontal meridian (Weymouth et al., 1928), the lower performance for vertical than for horizontal words suggests that visual acuity is a major determinant of this phenomenon.

However, the cause of the asymmetry of the OVP curves cannot yet be determined from the results of Experiment 1 . Given that the curves were asymmetric toward the word beginning for both vertical and horizontal words, one could argue that the asymmetry might be caused by factors that affect both display conditions in the same way. The distribution of lexical information and factors that are related to the way information is extracted from print are possible candidates. In fact, post hoc analyses revealed that the words used in Experiment 1 were more informative at the first half than at the second half. The average number of words sharing the first trigram with a stimulus word was 4.19 , whereas the average number of words sharing the last trigram with a target word was $10.89[F(1,99)=28.92, p<.0001]$. When directly fixated, this informative first half of the word could enhance word identification performance and may bring the OVP toward the word beginning. Alternatively, the directional aspect of reading may favor processing of the beginning of words and, by virtue of this, enhance performance when the first half of a word is fixated.

The asymmetry of OVP could also be caused by factors that affect the two display conditions differently. The marked asymmetry for horizontal rather than for vertical words is not predicted by lexical factors or by the way in- formation is encoded from print. One possibility is that the pronounced asymmetry of the OVP curves for horizontal words comes from the previously mentioned hemispheric differences in the processing of language (Brysbaert et al., 1996). This hypothesis predicts a leftward asymmetry of the OVP curve for horizontal words, but no asymmetry for vertical words. The results of Experiment 1 concerning vertical words are inconsistent with this prediction, indicating that an asymmetry of the OVP is not determined by the laterality of language processing

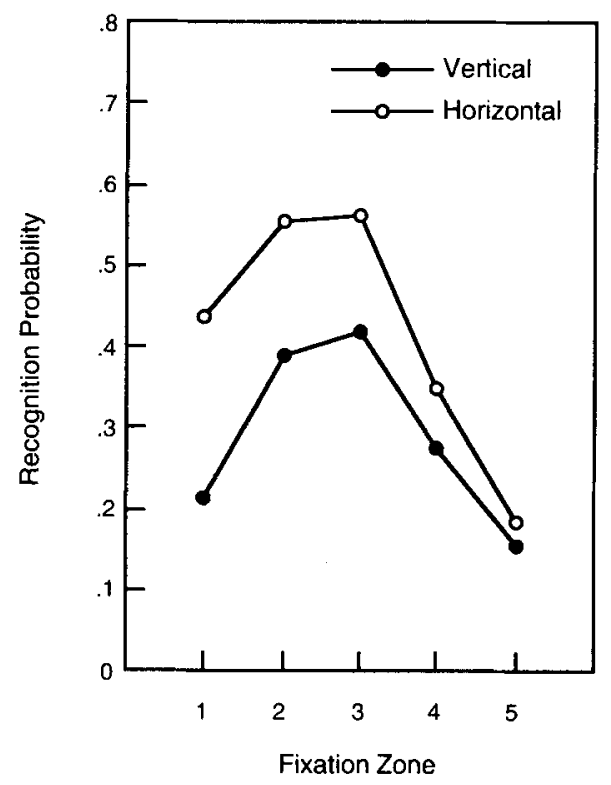

Figure 3. Word recognition probability as a function of fixation zone. The parameter indicates the presentation condition of the stimulus words. 
alone. Combined with the factors mentioned earlier, however, a lateralization view might account for the difference in the asymmetry for horizontal and vertical words.

Finally, it is also possible that simple variations in the visibility of letters, as was proposed by Nazir et al. (1991), will explain the obtained results. To account for the present results in terms of character visibility, assuming no lexical influence, the following conditions must be met. (1) Identification performance for individual characters should decrease linearly as the distance of the character from the center of vision increases. This should be valid for both the vertical and the horizontal meridians. (2) The drop-off rate of character visibility should be higher in the left visual field than in the right visual field. (3) The drop-off rate of character visibility should be higher for the upper than for the lower side of the fixation. (4) The left/right asymmetry along the horizontal meridian should be more marked than the upper/lower asymmetry along the vertical meridian. Experiment 2 served to verify these assumptions.

\section{EXPERIMENT 2}

To estimate variations in the visibility of characters in strings as a function of their location in the visual field, the probability of identifying a character flanked by two digits was determined along the vertical and the horizontal meridians. Digits were chosen as flankers, instead of characters, because a combination of two Hiragana characters often forms a word.

\section{Method}

Subjects. Eight right-handed graduate students, who were native Japanese with normal or corrected-to-normal vision, participated in the experiment. Five of them had also participated in Experiment 1.

Material. Five Japanese Hiragana characters were used as target stimuli. The flanking stimulus was the digit 8 . The sizes of the characters and of the digit were equal and were $0.6^{\circ}$ in visual angle.

Design. The stimulus string was displayed at six different eccentricities (a space of zero to five characters) either above or below the fixation point (vertical condition), or left or right of the fixation point (horizontal condition). In the vertical display condition, the flankers were placed above and below the target; in the horizontal display condition, they were placed left and right of the target. The experiment contained 660 trials for each subject. The target stimulus with the flanking stimuli appeared six times at each possible location in the visual field. The position of presentation (upper or lower, left or right) and eccentricity were randomized.

Procedure. The subjects placed their heads on a chinrest. In each trial, the subject was instructed to fixate on the center of the fixation mark that consisted of four diagonal lines (see Figure 4). The fixation lines remained for $500 \mathrm{msec}$. Then the stimulus string was presented for $71 \mathrm{msec}$ and was replaced by a cross consisting of 21 hashmarks, which remained on the screen for $300 \mathrm{msec}$. The subject's task was to identify the target character and to type it on the keyboard in Roman letters. Twenty-five practice trials were given before starting the main experimental session.

\section{Results}

An ANOVA showed significant main effects of presentation side $[F(3,21)=20.43, p<.001]$ and eccentric- ity $[F(4,28)=82.63, p<.001]$. The interaction between these two factors was also significant $[F(12,84)=4.60$, $p<.001]$. As can be seen in Figure 5, identification performance decreases linearly for the vertical meridian, as well as for the horizontal.

A regression analysis was performed for each subject by adjusting the intercept for the four regression lines (above, below, left, and right conditions) and by minimizing the sum of squared deviations between theoretical lines and the empirical data. The mean slope and $R^{2}$ of the regression lines for the four sides of the fixation point are shown in Table 1. A significant linear trend was found for each of the 4 regression lines for each single subject ( $p<.05$, for each of 32 regression lines). The probability of identifying a character dropped more rapidly on the left side of the fixation point than on the right side $[F(1,7)=11.54, p<.02]$. However, the drop-off rate for the upper side did not significantly differ from that for the lower side.

\section{Discussion}

Experiment 2 showed that for all of the four conditions, identification scores for characters in a string decrease linearly as the eccentricity of the target from fixation increases. These results correspond to the fact that the visual acuity decreases linearly for both the vertical and the horizontal meridians (Weymouth et al., 1928). For the vertical meridian, the drop-off rate of performance did not differ between positions above and below the fixation point. For the horizontal meridian, however, the drop-off rate was stronger on the left side than on the right side, consistent with previous results with the Roman script (Bouma, 1973; Bouma \& Legein, 1977; Nazir et al., 1992; but no hemifield differences were found by Bouma, 1970). Note that with these results, three of the four conditions described above were met.

To test more directly whether the visibility of characters can account for the viewing position effect in words, we calculated theoretical OVP curves for vertical and horizontal words, on the basis of the empirical results of Experiments 1 and 2, with the following equation:

$$
\begin{aligned}
P_{\text {word }}\left(f, a, b_{\text {left }}, b_{\text {right }}\right)= & \prod_{n=1}^{f}\left(a-(1.2 \cdot f-n-0.1) \cdot b_{\text {left }}\right) \\
& \cdot \prod_{n=f+1}^{6}(a-(-1.2 \cdot f+n+0.1) \\
& \left.\cdot b_{\text {right }}\right),
\end{aligned}
$$

where $P_{\text {word }}\left(f, a, b_{\text {left }}, b_{\text {right }}\right)$ is the probability of recognizing a word as a function of $f$, the relative fixation zone (from 1 to 5), $a$, the probability of recognizing the directly fixated character, and $b_{\mathrm{left}}$ and $b_{\text {right }}$, the rates of decrease in the probability of recognizing a character with increasing eccentricity, going left- or rightward. The formula is essentially based on Nazir et al. (1998), with slight modifications in order to apply their equations to the present 


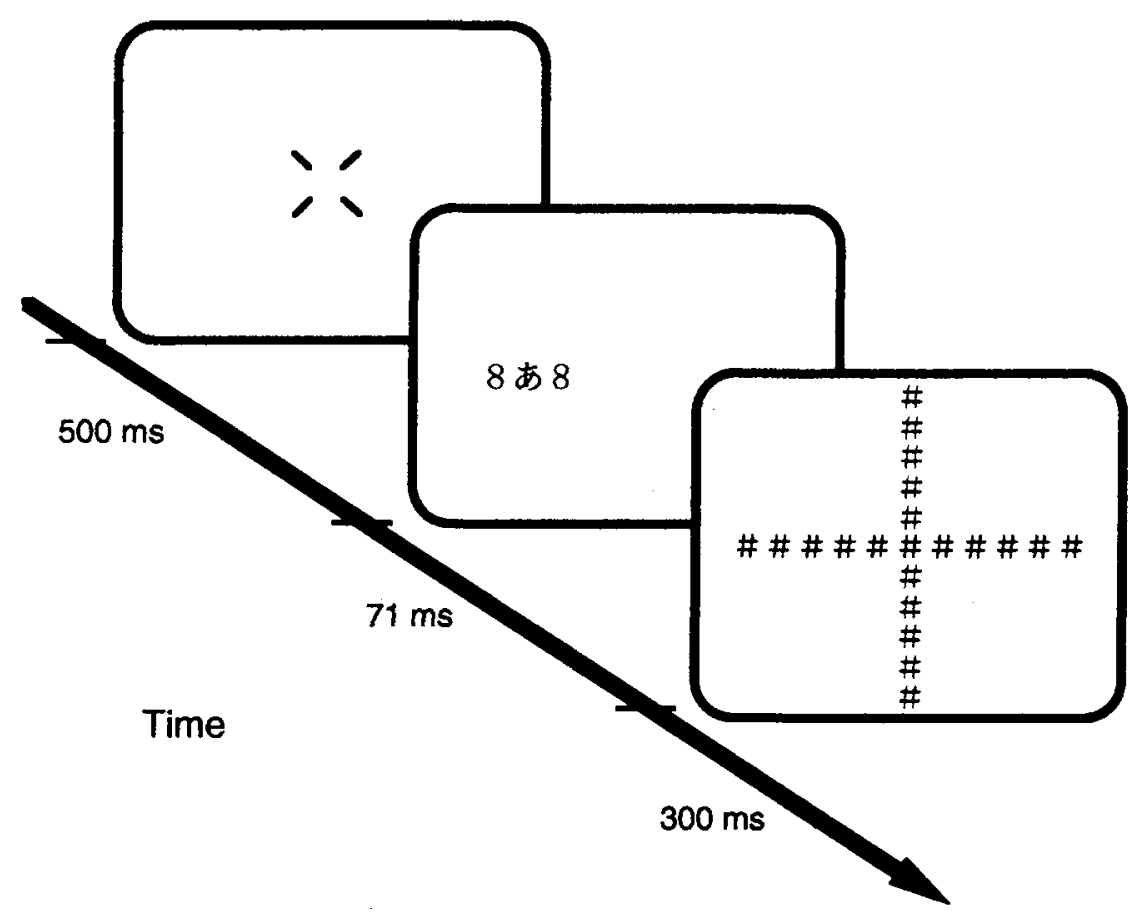

Figure 4. Time course and configuration of the stimulus presentation in Experiment 2.

study. Both $(1.2 f-n-0.1)$ and $(-1.2 f+n+0.1)$ indicate the distance between the center of the $n$th character in the word and the fixation position, where 1.2 and 0.1 were used to transform the five zone-based positions into the six character-based positions. When Equation 1 is applied to the performance for vertical words, we replaced the parameter $b_{\text {left }}$ with $b_{\text {upper }}$ and the parameter $b_{\text {right }}$ with $b_{\text {lower }}$. With parameter $a=1$ and the empirically obtained ratio of $b_{\text {left }}: b_{\text {right }}: b_{\text {upper }}: b_{\text {lower }}=0.119: 0.089: 0.150$ $: 0.148$ (see Table 1), we estimated the value of parameter $b_{\text {right }}$ from the empirical OVP curves for vertical and for horizontal words in Figure 3. For a minimum summed squared deviation $(S S D)=0.028$ between the theoretical and the empirical curves, the $b_{\text {right }}=0.056$.

Figure 6 (left panel) shows the theoretical OVP curves for vertical and horizontal words, together with the empirical data. The theoretical curves are similar to the empirical curves in the following points. First, the curve for vertical as well as for horizontal words describes an inverted-U-shaped function. Second, the curve for horizontal words is asymmetric to the word beginning. Third, the difference in height between the two curves is larger for the first half of the word than for the second half. On the other hand, the following differences were found between the theoretical and the empirical curves. First, the theoretical curve for vertical words was symmetric, whereas the empirical curve was asymmetric to the word beginning. Second, the two theoretical curves globally differed in height, whereas the two empirical curves were quite close to each other at the fourth and fifth fixation positions. Thus, character visibility accounts for only part of the OVP phenomenon, and other factors need to be considered. We will discuss these issues in the General Discussion section.

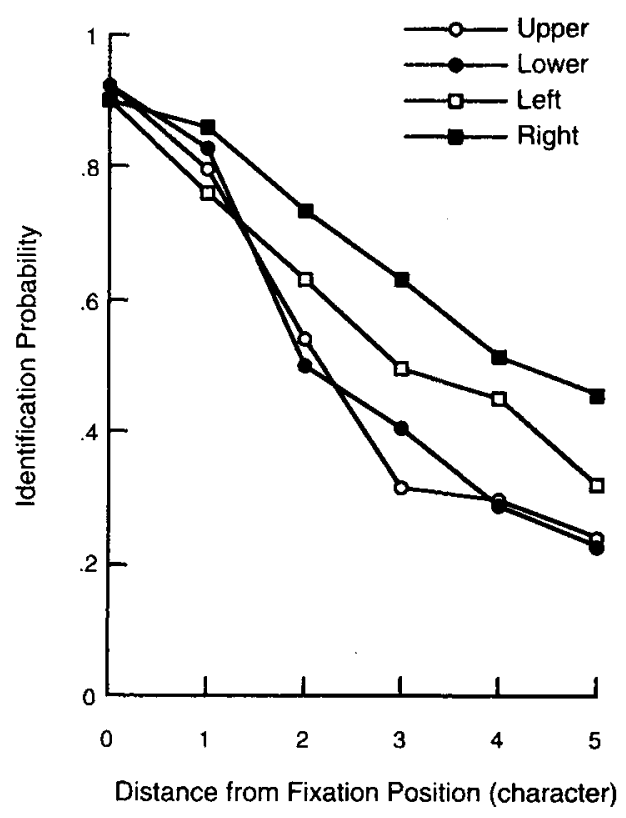

Figure 5. Identification probability of characters with flanking noise as a function of distance from the center of the visual field in character units. The parameter indicates the presentation side of the stimulus character. 
Table 1

Coefficients of Regression Lines

\begin{tabular}{ccc}
\hline $\begin{array}{c}\text { Position of } \\
\text { Target Character }\end{array}$ & Mean Slope & Mean $R^{2}$ \\
\hline Upper & -0.150 & .88 \\
Lower & -0.148 & .87 \\
Left & -0.119 & .83 \\
Right & -0.089 & .77 \\
\hline
\end{tabular}

\section{GENERAL DISCUSSION}

In the present study, we examined the OVP phenomenon in vertically and horizontally presented Japanese words. In particular, the model of Nazir et al. (1991), according to which the phenomenon is related to the visibility of letters, was directly tested. We found that the model can account for the inverted U-shape of the OVP curves that we observed for vertical as well as for horizontal words and for the asymmetry of the curve for horizontal words. It also offers an explanation of why the asymmetry of the OVP curve is more marked for horizontal than for vertical words. However, the model did not account for the asymmetry of the OVP curve for vertical words. Thus, although letter visibility seems to play a role, this factor is not sufficient to explain the entire phenomenon.

To better account for the empirical results, it seems that a mathematical model of the OVP phenomenon would have to include at least two other factors and describe their mutual relation. This will be proposed in the following.

In a first step, we offer a model in which letter visibility and lexical factors are combined. In short, lexical factors are added to the original model. As was pointed out by Nazir et al. (1991), their model considers only visual factors. Word recognition is accomplished only when all letters of the word are successfully encoded by the visual system. However, it is well known that a word can be recognized from a limited amount of sensory information, using lexical knowledge (Brysbaert et al., 1996; Farid \& Grainger, 1996; McClelland \& Rumelhart, 1981). Here, we consider the case in which a six-letter word is identified with information extracted from its first or last half-namely, its first or last trigram. When the first trigram of the word is successfully encoded, but not its last trigram, the probability of recognizing the word will depend on the probability of correctly inferring the word from the information about this first trigram and can be estimated with the following equation:

$$
P_{\text {word (first) }}=P_{\text {first }} \cdot\left(1-P_{\text {last }}\right) \cdot Q_{\text {first }} \text {, }
$$

where $P_{\text {word (first) }}$ is the probability of recognizing the word from the first trigram, $P_{\text {first }}$ and $P_{\text {last }}$ are the probabilities of successfully encoding the first and last trigrams, respectively, and $Q_{\text {first }}$ is the probability of correctly guessing the word from the encoded information about the first trigram. Note that $P_{\text {first }}$ and $P_{\text {last }}$ are estimated from the same parameters of Equation 1 (see Equations 3 and 4 , at the bottom of this page). The right part of Equation 1 , therefore, is equal to the product of $P_{\text {first }}$ and $P_{\text {last }}$. When the visual system successfully encodes the last trigram, but not the first trigram, the probability of recognizing the six-letter word is estimated with the following equation:

$$
P_{\text {word(last) }}=\left(1-P_{\text {first }}\right) \cdot P_{\text {last }} \cdot Q_{\text {last }},
$$

where $P_{\text {word(last) }}$ is the probability of recognizing a word from the last trigram and $Q_{\text {last }}$ is the probability of correctly guessing the word from the encoded information about the last trigram.

If lexical knowledge facilitates performance, word recognition probability can be expressed as the sum of Equations 1, 2, and 5:

$$
\begin{gathered}
P_{\text {word }}\left(f, a, b_{\text {left }}, b_{\text {right }}, Q_{\text {first }}, Q_{\text {last }}\right)=P_{\text {first }} \cdot P_{\text {last }}+P_{\text {first }} \\
\cdot\left(1-P_{\text {last }}\right) \cdot Q_{\text {first }}+\left(1-P_{\text {first }}\right) \cdot P_{\text {last }} \cdot Q_{\text {last }} .
\end{gathered}
$$

To determine whether Equation 6 can describe the results of the present study, we calculated the corresponding theoretical OVP curves. Visual parameters were assumed to be the same as those in the previous calculation: Parameter $a=1$, and the ratio of $b_{\text {left }}: b_{\text {right }}: b_{\text {upper }}$ : $b_{\text {lower }}=0.119: 0.089: 0.150: 0.148$. The reciprocal of the number of the words that share the same trigram as

$$
P_{\text {first }}= \begin{cases}\prod_{n=1}^{f}\left(a-(1.2 \cdot f-n-0.1) \cdot b_{\text {lefi }}\right) \cdot \prod_{n=f+1}^{3}\left(a-(-1.2 \cdot f+n+1) \cdot b_{\text {right }}\right) & (f \leq 2) \\ \prod_{n=1}^{3}\left(a-(1.2 \cdot f-n-0.1) \cdot b_{\text {left }}\right) & (f \geq 3)\end{cases}
$$

and

$$
P_{\text {last }}= \begin{cases}\prod_{n=4}^{6}\left(a-(-1.2 \cdot f+n+0.1) \cdot b_{\text {right }}\right) & (f \leq 3) \\ \prod_{n=4}^{f}\left(a-(1.2 \cdot f-n-0.1) \cdot b_{\text {left }}\right) \cdot \prod_{n=f+1}^{6}\left(a-(-1.2 \cdot f+n+0.1) \cdot b_{\text {right }}\right) & (f \geq 4) .\end{cases}
$$



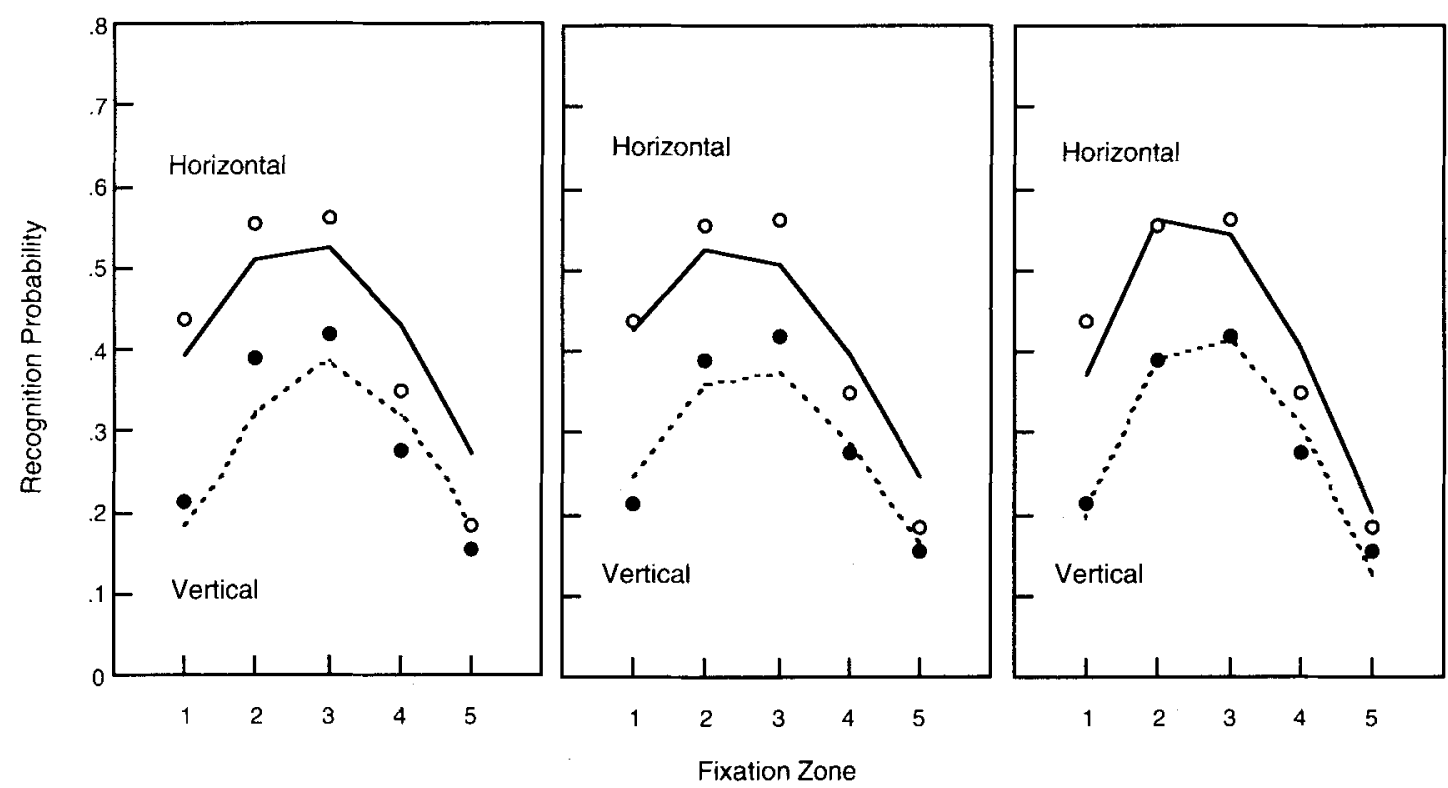

Figure 6. Theoretical and empirical optimal viewing position (OVP) curves as a function of fixation zone. Lines indicate the theoretical curves. Circles indicate the empirical data. Continuous lines and open circles correspond to the horizontal condition, whereas dotted lines and closed circles correspond to the vertical condition. The theoretical curves are estimated following the multiplied letter identification probability model (left panel), a model in which visual and lexical factors are combined (middle panel), and a model in which visual and lexical factors are combined and the endletter superiority effect is considered (right panel).

the stimulus words was used as an approximation for the lexical parameters: $Q_{\text {first }}=.25$ and $Q_{\text {last }}=.1$ (reciprocals of 4 and 10 , respectively). Parameter $b_{\text {right }}$ was estimated from the empirical OVP curves both for vertical and for horizontal words (Experiment 1). For a minimum SSD of $0.014, b_{\text {right }}=0.072$. Note that the estimated $b_{\text {right }}$ is now closer to the empirical value of 0.089 than is the previous estimation of 0.056 , following the original model (see the Discussion section of Experiment 2). In addition, the $S S D$ is smaller than that in the previous estimation, 0.028 , which indicates that the present model explains the OVP phenomenon better than does the original model. In fact, consistent with the empirical results, the theoretical OVP curves are now asymmetric to the word beginning for vertical as well as for horizontal words (Figure 6 , middle panel). Thus, adding lexical factors provides a more precise description of the observed empirical OVP phenomenon.

The model described above can be further improved by modifying the estimations of the visual parameters $P_{\text {first }}$ and $P_{\text {last }}$ and the lexical parameters $Q_{\text {first }}$ and $Q_{\text {last }}$. The first modification concerns the issue of whether a simple linear function should be used to estimate the probability of correctly encoding letters in a word. Since a letter is more easily recognized when it is flanked on one side than when flanked on both sides (Bouma, 1970), letters located at the end of a string are more visible than is predicted by the linear function that is currently used. In fact, the letter-in-string experiment by Nazir et al. (1991) showed that the identification probability of the most peripheral letter is much higher than that of the second and seventh letters in an eight-letter string. This superiority of the end letters was also observed in Nazir et al. (1998) for five- and nine-letter strings, and its significance for word recognition was recently shown by Clark and O'Regan (1999). It is clear that $P_{\text {first }}$ and $P_{\text {last }}$ are affected by this end-letter superiority effect. To give a first estimation of how this effect could modulate the OVP curve, we simply assumed that the probability of correctly encoding the initial and the final letters in a word is identical to the probability of recognizing the letter immediately adjacent to the initial or the final letter of the string, although in reality, the visibility of the first and the last letters is probably underestimated by this assumption (Nazir et al., 1991). Following Equation 6, theoretical OVP curves were calculated with the same parameters as those for the above calculation. The results are shown in Figure 6 (right panel). The theoretical OVP curves became closer to the empirical curves, as is indicated by an $S S D=$ 0.010 . In addition, $b_{\text {right }}$ was estimated as 0.085 , which is almost identical to the empirical value of 0.089 . Thus, by including the superiority of end letters, the model seems to provide a fairly precise description of the OVP phenomenon.

The second modification concerns the issue of whether it is appropriate to estimate $Q_{\text {first }}$ and $Q_{\text {last }}$ by the recip- 


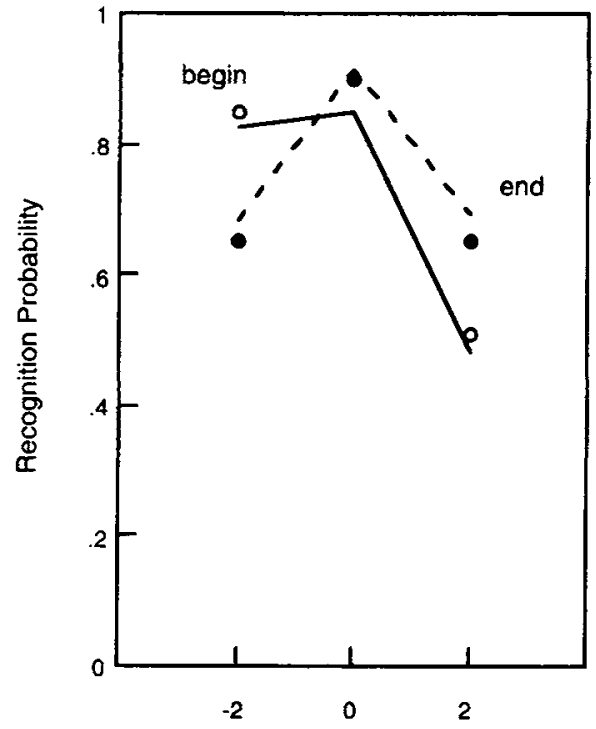

Fixation Position Relative to Word Center(character)

Figure 7. Theoretical and empirical optimal viewing position curves as a function of fixation position. Lines indicate theoretical curves, following a model in which visual and lexical factors are combined and the end-letter superiority effect is considered. Circles indicate empirical data from Brysbaert, Vitu, and Schroyens (1996). The continuous line and open circles correspond to words informative at the beginning, whereas the dotted line and closed circles correspond to words informative at the end.

rocal of the number of words that share the same trigram as the stimulus words. A more direct way of estimating these parameters would come from a word completion task in which subjects are asked to guess a word from its first or last trigram (see Brysbaert et al., 1996).

In fact, our model can well explain the results reported by Brysbaert et al. (1996) for their Experiment 3. For this, estimations of $Q_{\text {first }}$ and $Q_{\text {last }}$ are taken from their word completion task. According to these estimations, $Q_{\text {first }}=.84$ and $Q_{\text {last }}=.09$ for words informative at the beginning, and $Q_{\text {first }}=.08$ and $Q_{\text {last }}=.74$ for words informative at the end. Given that their data concern the Roman script, the values for parameter $b_{\text {left }} / b_{\text {right }}$ were set to $0.11 / 0.06=1.83$, as was indicated by Nazir et al. (1991), instead of our estimation using Japanese script. Using these parameters, we estimated the theoretical curves following from Equation 6 (adapted for fivecharacter words). As is demonstrated in Figure 7, for a drop-off rate of $b_{\text {right }}=0.0479$, the theoretical curves fit the Brysbaert et al. data, with $S S D=0.0075$.

To conclude, the OVP phenomenon in recognition accuracy for briefly presented words is likely to result from a combination of visual and lexical factors. The model we proposed explained well the OVP effect for vertical and horizontal words in Japanese, as well as for words in the Roman script. This model also has the potential to account for the small or even absent lexical effect on the asymmetry of OVP, such as that reported in the previous studies on Roman script. As is obvious from Equation 6, the contribution of lexical factors varies, depending on visual factors: The stronger the left/right asymmetry in the visibility of letters, the less obvious the lexical contribution will be.

\section{REFERENCES}

Bouma, H. (1970). Interaction effects in parafoveal letter recognition. Nature, 226, 177-178.

BoumA, H. (1973). Visual interference in the parafoveal recognition of initial and final letters of words. Vision Research, 13, 767-782.

Bouma, H. \& LEGEIN, C. P. (1977). Foveal and parafoveal recognition of letters and words by dyslexics and by average readers. Neuropsychologia, 15, 69-80.

Brysbaert, M., \& D'Ydewalle, G. (1988). Callosal transmission in reading. In G. Luer, U. Lass, \& J. Shallo-Hoffmann (Eds.), Eye movement research: Physiological and psychological aspects (pp. 246266). Göttingen: Hogrefe.

Brysbaert, M. Vitu, F., \& Schroyens, W. (1996). The right visual field advantage and the optimal viewing position effect: On the relation between foveal and parafoveal word recognition. Neuropsychologv, 10, 385-395.

ClaRk, J. J., \& O'Regan, J. K. (1999). Word ambiguity and the optimal viewing position in reading. Vision Research, 39, 843-857.

DEUTSCH, A., \& RAYNeR, K. (1999). Initial fixation location effects in reading Hebrew words. Language \& Cognitive Processes, 14, 393421 .

FARID, M., \& Grainger, J. (1996). How initial fixation position influences visual word recognition: A comparison of French and Arabic. Brain \& Language, 53, 351-368.

McClelland, J. L., \& Rumelmart, D. E. (1981). An interactive activation model of context effect in letter perception: Pt. I. An account of basic findings. Psychological Review, 88, 375-407.

MCConkie, G. W., Kerr, P. W., Rendix, M. D., Zola, D., \& Jacobs, A. M. (1989). Eye movement control during reading: II. Frequency of refixating a word. Perception \& Psychophysics, 46, 245-253.

Nazir, T. A., Heller. D., \& Sussmann, C. (1992). Letter visibility and word recognition: The optimal viewing position in printed words. Perception \& Psvchophwsics, 52, 315-328.

Nazik. T. A., Jarobs, A. M., \& O'Regan, J. K. (1998). Letter legibility and visual word recognition. Memory \& Cognition, 26, 810-821.

NAZiR, T. A.. O'Regan, J. K.. \& JaCOBS, A. M. (1991). On words and their letters. Bulletin of the Psychonomic Society, 29, 171-174.

O'Regan, J. K., \& JaCOBS. A. M. (1992). Optimal viewing position effect in word recognition: A challenge to current theory. Journal of Experimental Psychologv: Human Perception \& Performance, 18. 185-197.

O'Regian, J. K., Lévy-Schoen, A., Pynte, J., \& Brugallètre, B. (1984). Convenient fixation location within isolated words of different length and structure. Journal of Experimental Psychology: Human Perception \& Performance, 10, 250-257.

RADACH, R., \& KEMPE, V. (1993). An individual analysis of initial fixation position in reading. In G. d'Ydewalle \& J. van Rensbergen (Eds.). Perception and cognition: Advances in eve movement research (pp. 213-215). Amsterdam: North-Holland.

RAYNER. K. (1979). Eye guidance in reading: Fixation locations within words. Perception, 8, 21-30.

RAYNER, K., \& Pollatsek, A. (1989). The psychology of reading. Hillsdale, NJ: Eribaum.

Rayner. K., Sereno. S. C., \& Raney, G. E. (1996). Eye movement control in reading: A comparison of two types of models. Journal of Experimental Psychology: Human Perception \& Performance, 22, 1188-1200.

TAYLOR, L., \& TAYlor, M. M. (1995). Writing and literacy in Chinese. Korean, and Japanese. Amsterdam: Benjamins. 
Underwood, G., Clews, S., \& Everatt, J. (1990). How do readers know where to look next? Local information distributions influence eye fixations. Quarterly Journat of Experimental Psychology, 42A, 39-65. VITU, F. (1991). The influence of parafoveal preprocessing and linguistic context on the optimal landing position effect. Perception \& Psychophysics, 50, 58-75.

Vitu, F., O'Regan, J. K., \& Mittau, M. (1990). Optimal landing position in reading isolated words and continuous texts. Perception \& Psychophysics, 47, 583-600.
Weymouth, F. W., Hines, D. C.. Acres, L. H., RaAf, J. E., \& WHEEIER, M. C. (1928). Visual acuity within the area centralis and its relation to eye movements and fixation. American Journal of Ophthalmology, 11, 947-960.

(Manuscript received September 8, 1998; revision accepted for publication January 27,2000 .) 